\title{
Efficient Algorithms for the Two Locus Problem in Genome-Wide Association Study
}

\author{
Sanguthevar Rajasekaran ${ }^{*}$ \\ Department of Computer Science and \\ Engineering \\ University of Connecticut, Storrs, CT \\ rajasek@engr.uconn.edu
}

\author{
Subrata Saha \\ Department of Computer Science and \\ Engineering \\ University of Connecticut, Storrs, CT \\ subrata.saha@engr.uconn.edu
}

\begin{abstract}
Advances made in sequencing technology have resulted in the sequencing of thousands of genomes. Novel analysis tools are needed to process these data and extract useful information. Such tools could aid in personalized medicine. As an example, we could identify the causes for a disease by comparing the genomes of people who have the disease and those who do not have this disease. Given that human variability happens due to single nucleotide polymorphisms (SNPs), we could focus our attention on these SNPs. Investigations that try to understand human variability using SNPs fall under genome-wide association study (GWAS). A crucial step in GWAS is the identification of the correlation between genotypes (SNPs) and phenotypes (i.e., characteristics such as the presence of a disease). This step can be modeled as the $k$-locus problem (where $k$ is any integer). A number of algorithms have been proposed in the literature for this problem when $k=2$. In this paper we present an algorithm for solving the 2-locus problem that is up to two orders of magnitude faster than the previous best known algorithms.
\end{abstract}

\section{Keywords}

Genome-wide association study; Two-locus problem; String correlations

\section{INTRODUCTION}

There are a number of ways in which any two human genomes can differ. Variations are largely due to the single nucleotide polymorphisms (SNPs in short) as well as deletions, insertions and copy number variations [6]. Any of these variations may result in alterations in an individual's traits, or phenotypes. A phenotype of interest can be anything from a disease risk to physical properties such as height. In genetic epidemiology, a genome-wide associ-

\footnotetext{
${ }^{*}$ Corresponding Author
}

Permission to make digital or hard copies of all or part of this work for personal or classroom use is granted without fee provided that copies are not made or distributed for profit or commercial advantage and that copies bear this notice and the full citation on the first page. Copyrights for components of this work owned by others than ACM must be honored. Abstracting with credit is permitted. To copy otherwise, or republish, to post on servers or to redistribute to lists, requires prior specific permission and/or a fee. Request permissions from permissions@acm.org.

CIKM'16, October 24-28, 2016, Indianapolis, IN, USA

(c) 2016 ACM. ISBN 978-1-4503-4073-1/16/10 . \$ $\$ 15.00$

DOI: http://dx.doi.org/10.1145/2983323.2983685 ation study (GWA study, or GWAS), also known as whole genome association study (WGA study, or WGAS), is an examination of many common genetic variants in different individuals to observe if any variant is associated with a trait. GWAS plays a major role in personalized medicine.

A lot of effort has been spent to identify mappings between phenotypical traits and genomic data. Due to the advent of next generation high throughput sequencing technologies, nowadays it is possible to study the genomic structure of individuals in detail. In GWAS, two different problems have been focused on. In single locus association study, researchers try to find out the association between phenotypical traits and individual SNPs. In two locus association study, the goal is to figure out the association between pairs of SNPs and phenotypical traits. A major task in this study is that of identifying the most correlated pair of SNPs. Two locus associations are also known as gene-gene interactions. Such interactions are believed to be major factors responsible for many complex phenotypical traits $[10,2,5,7,8$, 14]. It is a proven fact that such gene-gene interactions are the major actors to express many complex traits such as various human diseases. A generalization of this problem is that of identifying the $k$ most important loci responsible for a specific phenotype. This is known as the $k$-locus problem.

Given that the number of SNPs found in humans is $10^{5}$ to $10^{7}$, a brute force way of scanning through every possible pair of SNPs to identify the most correlated pair is not feasible in practice. A number of algorithms for the two locus problem can be found in the literature. For instance, genetic algorithms are used in [11] and [13]. The algorithms proposed in [15] and [16] take $O\left(n^{2} m+n m^{2}\right)$ time, where $n$ is the number of SNPs and $m$ is the number of subjects. This algorithm is called FastANOVA. An algorithm with an expected run time of $O\left(n^{1+\epsilon} \log ^{2} n\right)$, where $0<\epsilon<1$ is a constant, has been presented in [1]. This algorithm exploits an algorithm known for the Light Bulb Problem [12] and Locality Sensitive Hashing (LSH) [3]. In this paper we present an algorithm for the two locus problem whose expected run time is $O\left(n^{1+\epsilon} \log n\right)$ (where $0<\epsilon<1$ is a constant). Our algorithm is asymptotically better than that of [1] by a logarithmic factor. Moreover, we have employed a number of innovations at the coding level and hence the improvement is indeed by more than a logarithmic factor. Our algorithm is up to two orders of magnitude faster than prior algorithms (specifically FastANOVA [16] and the algorithm of [1]) on various benchmark datasets.

The rest of this paper is organized as follows. In Section 2 we provide some notations. In Section 3 we present 
some preliminaries needed for solving the two locus problem. Specifically, we consider the problems of finding the largest correlated pair of strings (from out of $n$ given strings) and the problem of finding the least correlated pair of strings. We present novel algorithms for solving these problems and compare them with prior best known algorithms for solving these. In Section 4 we describe our algorithm (called TLA) for solving the two locus problem and in Section 5 we compare our algorithm with prior best algorithms. Some concluding remarks are given in Section 6 .

\section{NOTATIONS AND DEFINITIONS}

Let $T=a_{1}, a_{2}, \ldots, a_{n}$ be a sequence of real numbers (or characters from a finite alphabet). An $\ell$-mer of $T$ is nothing but a subsequence of $T$ of $\ell$ contiguous elements of $T$. The $\ell-$ mers of $T$ are $T_{i}=a_{i}, a_{i+1}, \ldots, a_{i+\ell-1}$, for $1 \leq i \leq(n-\ell+1)$.

If the elements of $T$ are real numbers, then the Euclidean distance between $T_{i}$ and $T_{j}$, denoted as $d\left(T_{i}, T_{j}\right)$, is $\sqrt{\sum_{k=0}^{\ell-1}\left(a_{i+k}-a_{j+k}\right)^{2}}$.

If the elements of $T$ are characters from an alphabet $\Sigma$, then the Hamming distance between $T_{i}$ and $T_{j}$, denoted as $d\left(T_{i}, T_{j}\right)$, is $\sum_{k=0}^{\ell-1} \delta\left(a_{i+k}, a_{j+k}\right)$ where $\delta(a, b)=1$ if $a \neq b$ and $\delta(a, b)=0$ if $a=b$ (for any $a, b \in \Sigma$ ). A sequence of characters can be thought of as a string of characters, since we can obtain a string from the sequence by concatenating the characters. Thus we'll use the terms 'a sequence of characters' and 'a string of characters' interchangeably.

Let $A=a_{1}, a_{2}, \ldots, a_{n}$ and $B=b_{1}, b_{2}, \ldots, b_{n}$ be two sequences of characters. Also, let the Hamming distance between $A$ and $B$ be $d$. Then, by the number of matches between $A$ and $B$ we mean $n-d$. Also, the correlation between $A$ and $B$ is defined to be $\frac{n-d}{n}$.

\section{SOME PRELIMINARIES}

In this section we provide some preliminary ideas and techniques that will be useful for solving the two locus problem. Specifically, in this section we consider the following problems: finding the most similar pair of character strings, and finding the least similar pair of character strings, where the similarity is in terms of the Hamming distance between strings.

\subsection{Finding the most correlated pair of strings}

For this problem we are given $n$ Boolean vectors $\hat{b}_{1}, \hat{b}_{2}, \ldots, \hat{b}_{n}$ each of length $t$. The problem is to find the pair of vectors that are the most similar (i.e., the Hamming distance between them is the smallest). Note that, given two vectors, we can find the Hamming distance between them in $O(t)$ time. A straight forward algorithm to identify the most correlated pair of vectors takes $O\left(n^{2} t\right)$ time. This algorithm computes the Hamming distance between every pair of vectors. We can achieve a better run time using randomization. We say that the correlation between a pair of strings is $p$ if the Hamming distance between them is $t(1-p)$. Let $p_{1}$ be the correlation between the most correlated pair of strings and $p_{2}$ be the correlation between the second most correlated pair of strings.

Consider a matrix $M$ of size $n \times t$, such that the $i$ th row of $M$ is $\hat{b}_{i}$, for $1 \leq i \leq n$. The idea of our algorithm is to iteratively collect pairs of strings that are candidates to be the most correlated. Once we collect enough pairs, we compute the distance between each pair in this collection and output the closest. In each iteration we pick $q$ columns randomly. For any vector (or string), the values in these columns can be concatenated to get a $q$-bit integer. We hash the vectors based this integer value. Subsequently, we generate pairs as follows: Consider any bucket in the hash table. If there are $m$ vectors in this bucket, then each pair of vectors in this bucket is added as a candidate to a list $C$. There are $O\left(n^{\frac{\log p_{1}}{\log p_{2}}} \log n\right)$ iterations in the algorithm.

We can show that after $O\left(n^{\frac{\log p_{1}}{\log p_{2}}} \log n\right)$ iterations, $C$ will have the most correlated pair of bulbs with high probability (i.e., with a probability of $1-n^{-\Omega(1)}$ ). This algorithm is similar to the algorithm given in [12] but faster (by more than a logarithmic factor). We can generalize the above algorithm to arbitrary alphabets and get the following

Theorem 3.1. Let $M$ be a matrix of size $n \times t$. Each entry in this matrix is an element from some set $\Sigma$ of cardinality $\sigma$. We can find the most correlated pair of rows of $M$ in an expected $O\left(n^{1+\frac{\log p_{1}}{\log p_{2}}} \log n\right)$ time where $p_{1}$ is the correlation between the most correlated pair of rows, $p_{2}$ is the correlation between the second most correlated pair of rows. (Here correlation is based on Hamming distance. For example, $p_{1}$ is the largest fraction of columns in which any two rows agree).

Proof: Probability that the most correlated pair of strings falls into the same bucket is $p_{1}^{q}$. (Note that each row is a string). Probability that this pair does not fall into the same bucket in a given iteration is $\left(1-p_{1}^{q}\right)$. Thus, the probability that this pair does not fall into the same bucket in $z$ successive iterations is $\left(1-p_{1}^{q}\right)^{z}$. This probability is $\leq \exp \left(-z p_{1}^{q}\right)$ using the fact that $(1-x)^{1 / x} \leq 1 / e$ for any $0<x<1$. In turn, this probability will be $\leq n^{-\alpha}$ if $z \geq \frac{\alpha \log n}{p_{1}^{q}}$.

To ensure that the run time is as small as possible, we want to ensure that the size of $C$ is not too large. Since we spend a linear time in each iteration of the algorithm, an optimal strategy will be to make sure that the number of candidate pairs generated in each iteration is also $O(n)$. If $p_{2}$ is the second largest correlation, the probability that any pair other than the largest correlated falls into the same bucket in any iteration is $\leq p_{2}^{q}$. If this probability is $\leq \frac{1}{n}$, then the expected number pairs generated in any iteration will be $\leq n$. This happens if $q=\frac{\log n}{\log \left(1 / p_{2}\right)}$. For this value of $q$, the value of $z$ becomes $\alpha \log n n^{\log p_{1} / \log p_{2}}$.

Given that the expected time we spend in hashing in each iteration and the time for generating the pairs is $O(n)$, it follows that the expected run time of the algorithm is $O\left(n^{1+\frac{\log p_{1}}{\log p_{2}}} \log n\right)$.

Note: The run time of MCP is better than that of the light bulb algorithm presented in [12] by more than a logarithmic factor.

Let the above general algorithm be called MCP.

The case of random data. To get an idea of how large $\frac{\log p_{1}}{\log p_{2}}$ could get consider the case where all the strings are random (i.e., each character of any string is randomly chosen to be 0 or 1 with equal probability). Consider a collection of $n$ random strings of length $t$ each. Let $A$ and $B$ be any 
two of these vectors. The expected correlation between $A$ and $B$ is $1 / 2$, i.e., the expected number of positions in which they match is $t / 2$. We can use Chernoff bounds to get high probability estimates on $p_{1}$ and $p_{2}$.

Chernoff bounds: If $X$ is the sum of $t$ independent Bernoulli trials with a probability $p$ of success each, then the following are true, for any $0<\delta<1[9]$ :

$$
\text { Prob. }\left[X<((1-\delta) \mu]<\exp \left(-\mu \delta^{2} / 2\right)\right. \text {. }
$$

Here $\mu=t p$. Also, if $p \geq 1 / 2$, then, for any $x>0$,

$$
\operatorname{Prob} .[X>t p+x]<\exp \left(\frac{-x^{2}}{2 t p(1-p)}\right) \text {. }
$$

Using the above bounds, it follows that the probability that the correlation between $A$ and $B$ is more than $\frac{t}{2}+x$ is $\leq$ $\exp \left(-2 x^{2} / t\right)$. Thus the probability that there exists at least one pair whose correlation is $>\frac{t}{2}+x$ is $\leq n^{2} \exp \left(-2 x^{2} / t\right)$. As a result, we infer that the correlation between any pair is $\leq \frac{1}{2}+\sqrt{\frac{2 \ln n-\ln u}{t}}$ with probability $\geq(1-u)$, for any $0<$ $u<1$. Similarly, the probability that none of the pairs has a correlation less than $\frac{1}{2}-\sqrt{\frac{2 \ln n-\ln u}{t}}$ is $\geq(1-u)$. Thus a high probability upper bound on $p_{1}$ is $\hat{p}_{1}=\frac{1}{2}+\sqrt{\frac{2 \ln n-\ln u}{t}}$ and a high probability lower bound on $p_{2}$ is $\check{p}_{2}=\frac{1}{2}-\sqrt{\frac{2 \ln n-\ln u}{t}}$.

Table 1 displays these estimates for different values of $n$. For this table, the value of $t$ has been chosen to be 200 and that of $u$ is 0.01 . It also shows the corresponding values of $\frac{\log \hat{p}_{1}}{\log \tilde{p}_{2}}$. From this table we see that for a fixed value of $t$ when the value of $n$ increases, the value of $\frac{\log \hat{p}_{1}}{\log \check{p}_{2}}$ decreases.

Table 1: Correlation estimates for the case of random data.

\begin{tabular}{llll}
\hline$n$ & $\hat{p}_{1}$ & $\check{p}_{2}$ & $\frac{\log \hat{p}_{1}}{\log \check{p}_{2}}$ \\
\hline 10,000 & 0.8393 & 0.1607 & 0.0958 \\
\hline 50,000 & 0.8622 & 0.1378 & 0.0748 \\
\hline 100,000 & 0.8717 & 0.1283 & 0.0669 \\
\hline
\end{tabular}

\subsection{An experimental comparison of $M K$ and MCP}

An algorithm called MK for solving the Time Series Motif Mining (TSMM) problem has been given in [4]. MK can be used to solve the problem of finding the most correlated pair of strings as well, since both of these problems are special cases of the well studied closest pair problem. The algorithm of [4] for approximate TSMM partitions the input time series data $T$ based on a window of size $w$ (for an appropriate value of $w$ ), computes the mean of every window, and discretizes the mean into four possible values. As a result, the time series data is transformed into a string $T^{\prime}$ of characters form the alphabet $\{1,2,3,4\}$. It then uses any $(\ell, d)$-motif finding algorithm to find the motifs in $T^{\prime}$. However, all the exact algorithms for finding $(\ell, d)$-motifs take time that is exponential on $\ell$ and $d$. Note that the last step of finding $(\ell, d)$ motifs can be replaced with a problem of finding time series motifs in $T^{\prime}$ which is nothing but the closest pair problem in the domain of strings of characters, the motif length being $\ell$.

One could employ MK to solve the problem of finding the most correlated pair of strings. The only difference is that we have to replace Euclidean distance with Hamming distance. We have implemented this algorithm. It turns out that MK does not perform well for the case of binary strings. To be fair, the authors of MK have not tested MK for this case. We have compared MK with MCP and the results are shown in Table 2. As this Table reveals, MCP has a much better performance than MK. Please, see Figure 1 for visual details. It is also clear that if we employ MCP in place of $(l, d)$-motif finding algorithms, the performance of the approximate TSMM algorithm given in [4] will improve significantly.

When $n$ is very large, we inject pairs with known correlations. As an example, consider the problem of finding the largest correlated pair of rows in a $m \times n$ matrix $M$. Say we generate each row by picking each element to be either 0 or 1 with equal probability. For any two rows, clearly, the expected correlation is $\frac{1}{2}$. We can perform a probabilistic analysis to get a high probability bound on the largest correlation between any two rows (see Table 1 ). For example, for $n=10,000$, we generated several random data sets and computed the largest correlation in each and calculated an average. Let $p$ be this value. To inject a pair with a correlation of $p^{\prime}$ where $p^{\prime}$ is $>p$ we generate a row $a$ with all ones and another row $b$ with $p^{\prime} m$ ones and $m\left(1-p^{\prime}\right)$ zeros. We then replace (any) two rows of $M$ with $a$ and $b$. Clearly, the correlation between $a$ and $b$ is $p^{\prime}$. The expected correlation between $a$ and any other row of $M$ (other than $b$ ) is $\frac{1}{2}$. Similarly, the expected correlation between $b$ and any other row of $M$ (other than $a$ ) is $\frac{1}{2}$. Thus the pair $(a, b)$ is likely to be the winner with high probability. We have picked a value for $p^{\prime}$ that is only slightly larger than $p$ so as to get an accurate estimate on the run time. We have used a similar technique to inject pairs to find most correlated pairs as well. We stop our algorithm MCP after a fixed number of runs i.e., 50. We are always able to find the most correlated pair we injected within the fixed number of runs. In Table 3 we show the results for our algorithm. As evident from Table $3 \mathrm{MCP}$ finds the injected pair within a very short period of time. As an example consider the dataset D3.8. It consists of 16 million timeseries, the motif length being 1024 . It processed $249.02 \times 10^{8}$ pairs only in 37 minutes to find the most correlated pair. Please, see Figure 1. Note that all the experiments were done on an Intel Haswell compute node with 48 GB of RAM. The operating system running was Red Hat Enterprise Linux Server release 5.7 (Tikanga).

\subsection{Identification of the Least Correlated Pair of Strings}

The MCP algorithm identifies the closest pair of strings, from out of $n$ given binary strings. An interesting question is if we can use the same algorithm to identify the furthest pair of strings. This problem has relevance in many problems including the two locus problem in GWAS. The authors of [1] present an elegant adaptation of the light bulb algorithm of [12] to solve this problem when the strings are binary. In this section we show how to adapt MCP to solve this problem. Our experimental comparison shows that our algorithm has a significantly better run time than that of [1].

\subsubsection{Some notations}

Let $m(x, y)$ stand for the number of matches between two strings (of equal length) $x$ and $y$. For instance, if $x=10010$ and $y=00111$, then $m(x, y)=2$ (since they match in posi- 
Table 2: Number of pairs and runtime comparisons in Hamming space on binary strings. CPU times are given in seconds.

\begin{tabular}{llllllll}
\hline & & MK & & MCP & & Gain & Speed up \\
\hline Dataset & Size & Processed Pairs & CPU Time & Processed Pairs & CPU Time & Processed Pairs & Time \\
\hline D2.1 & $2 \times 10^{3}$ & 976,520 & 2.34 & 1,515 & 0.27 & 644.57 & 8.82 \\
\hline D2.2 & $4 \times 10^{3}$ & $3,437,171$ & 9.09 & 6,099 & 0.44 & 563.56 & 20.81 \\
\hline D2.3 & $6 \times 10^{3}$ & $5,958,494$ & 17.50 & 13,889 & 0.51 & 429.01 & 33.99 \\
\hline D2.4 & $8 \times 10^{3}$ & $7,513,580$ & 22.51 & 24,308 & 0.80 & 309.10 & 28.29 \\
\hline D2.5 & $10 \times 10^{3}$ & $20,788,832$ & 60.59 & 38,076 & 0.81 & 545.98 & 74.69 \\
\hline D2.6 & $12 \times 10^{3}$ & $29,722,565$ & 90.28 & 54,707 & 0.95 & 543.30 & 94.87 \\
\hline D2.7 & $14 \times 10^{3}$ & $35,408,500$ & 110.38 & 75,058 & 1.09 & 471.75 & 101.08 \\
\hline D2.8 & $16 \times 10^{3}$ & $42,735,676$ & 130.22 & 97,934 & 1.26 & 436.37 & 103.06 \\
\hline D2.9 & $18 \times 10^{3}$ & $48,137,187$ & 157.73 & 124,126 & 1.37 & 387.81 & 114.90 \\
\hline D2.10 & $20 \times 10^{3}$ & $119,362,627$ & 381.11 & 152,837 & 1.51 & 780.98 & 251.86 \\
\hline
\end{tabular}

Table 3: Number of pairs and runtime of MCP in Hamming space on binary strings. CPU times are given in minutes.

\begin{tabular}{llllll}
\hline & & Brute-force & MCP & & Gain \\
\hline Dataset & Size & Processed Pairs & Processed Pairs & CPU Time & Processed Pairs \\
\hline D3.1 & $2 \times 10^{6}$ & $2 \times 10^{12}$ & $3.89 \times 10^{8}$ & 2.22 & $5,140.10$ \\
\hline D3.2 & $4 \times 10^{6}$ & $8 \times 10^{12}$ & $15.56 \times 10^{8}$ & 5.21 & $5,139.87$ \\
\hline D3.3 & $6 \times 10^{6}$ & $18 \times 10^{12}$ & $35.02 \times 10^{8}$ & 9.38 & $5,140.06$ \\
\hline D3.4 & $8 \times 10^{6}$ & $32 \times 10^{12}$ & $74.46 \times 10^{8}$ & 16.07 & $4,297.45$ \\
\hline D3.5 & $10 \times 10^{6}$ & $50 \times 10^{12}$ & $97.27 \times 10^{8}$ & 18.80 & $5,140.10$ \\
\hline D3.6 & $12 \times 10^{6}$ & $72 \times 10^{12}$ & $140.08 \times 10^{8}$ & 24.42 & $5,140.02$ \\
\hline D3.7 & $14 \times 10^{6}$ & $98 \times 10^{12}$ & $190.66 \times 10^{8}$ & 30.91 & $5,140.03$ \\
\hline D3.8 & $16 \times 10^{6}$ & $128 \times 10^{12}$ & $249.02 \times 10^{8}$ & 37.98 & $5,140.09$ \\
\hline
\end{tabular}

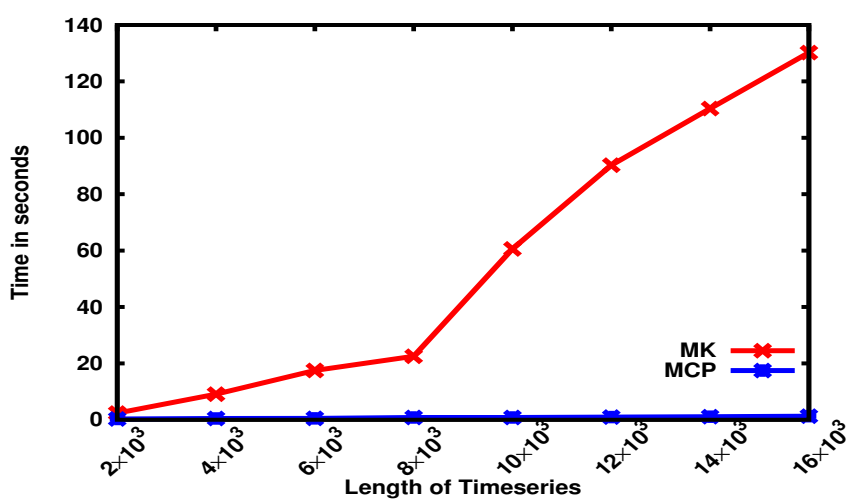

(a) CPU times consumed by MK and MCP methods

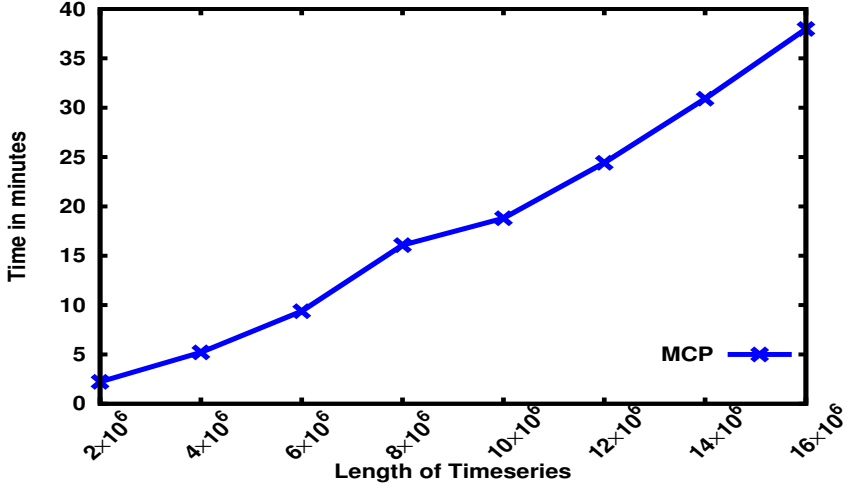

(b) CPU times consumed by MCP method

Figure 1: Performance evaluations between MK and MCP methods.

tions 2 and 4$)$. Let $X=x_{1}, x_{2}, \ldots, x_{q}$ and $Y=y_{1}, y_{2}, \ldots, y_{q}$ be two sequences of strings (each string having the same length). We define $M(X, Y)$ to be $\sum_{i=1}^{q} m\left(x_{i}, y_{i}\right)$.

Consider the sequences $A_{i}=a_{1}^{i}, a_{2}^{i}, \ldots, a_{k}^{i}$, for $1 \leq i \leq n$, where each $a_{j}^{i}$ is 0 , or 1 (for $1 \leq j \leq k$ ). Note that each $A_{i}$ is a sequence of strings where each string is of length 1 . Let $M\left(A_{i}, A_{j}\right)=u_{i j}$.

Each $A_{i}$ can be thought of as a binary string. In the application of GWAS, we can let $A_{i}$ correspond to the SNP $i$, for $1 \leq i \leq n$. Specifically, $a_{j}^{i}$ is the value of the $i$ th SNP in subject $j$, for $1 \leq j \leq k$. If we are interested in finding the two most correlated SNPs, then we can use MCP to identify this pair (as shown in Section 3.1). On the other hand, if our goal is to identify the least correlated pair, then, it is not clear how to do this using MCP. To solve the two locus GWAS problem, we have to identify not only the most correlated pair of strings but also the least correlated pair.

\subsubsection{Finding the least correlated pair}

The authors of [1] present an elegant solution for this problem. The idea is to construct a matrix $D$ of size $k \times 2 n$ where each column of $D$ corresponds to either a light bulb or its 'complement'. (A light bulb can be thought of as a string or a SNP). Specifically, the first $n$ columns correspond to the bulbs and the next $n$ columns correspond to the complements of the bulbs. In other words, $D[j, i]=a_{j}^{i}$, for $1 \leq i \leq$ $n, 1 \leq j \leq k$ and $D[j, i]=\bar{a}_{j}^{i}$ for $1 \leq j \leq k,(n+1) \leq i \leq 2 n$. Here, if $x$ is any bit, then, $\bar{x}$ denotes its complement. Let $D_{1}=\{q: 1 \leq q \leq n\}$ and $D_{2}=\{q:(n+1) \leq q \leq 2 n\}$. The algorithm of [1] for finding the least correlated pair works as follows. Consider all the pairs of columns $(a, b)$ such that 
$a \in D_{1}$ and $b \in D_{2}$. From out of these pairs, identify the pair $\left(a^{\prime}, b^{\prime}\right)$ of columns with the maximum number of matches. If $a^{\prime}=i$ and $b^{\prime}=n+j$, then $(i, j)$ is the least correlated pair of bulbs. Finding such a pair $\left(a^{\prime}, b^{\prime}\right)$ can be done using the light bulb algorithm of [12]. The correctness of this algorithm follows from the fact that if the two bulbs $i$ and $j$ have the least number of matches, then, column $i$ and the complement of column $j$ will have the most number of matches. In this approach we can replace the light bulb algorithm with MCP to drastically improve the run time (by more than a logarithmic factor).

\section{TWO LOCUS ASSOCIATION PROBLEM}

The two locus association problem is defined as follows. Input is a matrix $M$ of size $\left(m_{1}+m_{2}\right) \times n$ where $m_{1}+$ $m_{2}$ is the number of patients (subjects) each with $n$ SNPs. Here $m_{1}$ is the number of cases and $m_{2}$ is the number of controls. The cases are of phenotype 1 and the controls are of phenotype 0 . Rows 1 through $m_{1}$ of $M$ correspond to cases. Let this submatrix be called $A$. Rows $m_{1}+1$ through $m_{1}+m_{2}$ of $M$ correspond to controls and let this submatrix be called $B$. Each column of $M$ corresponds to an SNP. The two locus association problem is to identify the pair of SNPs whose statistical correlation with phenotype is maximally different between cases and controls. As mentioned in [1], the goal is to identify the pair:

$$
\underset{i, j}{\arg \max }\left|P_{A}(i, j)-P_{B}(i, j)\right| .
$$

If $Q$ is any matrix, then, $P_{Q}(i, j)$ stands for the correlation between the columns $i$ and $j$ of $Q$.

The algorithm of [1] exploits the light bulb algorithm of [12] and locality sensitive hashing (LSH) [3]. They use LSH to transform matrices $A$ and $B$ to $A^{\prime}$ and $B^{\prime}$, respectively. In particular, each column $c_{i}$ of $A$ is converted to a column $c_{i}^{\prime}$ of zeros and ones. The size of $c_{i}$ is $1 \times m_{1}$ and the size of $c_{i}^{\prime}$ is chosen to be $u=\max \left\{m_{1}, m_{2}\right\}$. The matrix $B$ is also transformed into $B^{\prime}$ in a similar manner using LSH. Followed by this, the pair of interest is identified.

To be precise, using $A^{\prime}$ and $B^{\prime}$, the matrix $D$ is formed where

$$
D=\left[\begin{array}{cc}
A^{\prime} & A^{\prime} \\
B^{\prime} & \bar{B}^{\prime}
\end{array}\right]
$$

where $\bar{B}^{\prime}$ is obtained from $B^{\prime}$ by complementing every element of $B^{\prime}$. Note that $D$ is of size $2 u \times 2 n$. Let $D_{1}=$ $\{1,2, \ldots, n\}$ and $D_{2}=\{n+1, n+2, \ldots, 2 n\}$. Consider all the pairs of columns $(i, j)$ such that $i \in D_{1}$ and $j \in D_{2}$. From out of these pairs, identify the pair $\left(i^{\prime}, j^{\prime}\right)$ of columns with the maximum number of matches. If $i^{\prime}=a$ and $j^{\prime}=n+b$, then $(a, b)$ is the pair of interest. The authors of [1] find this pair using the light bulb algorithm of [12].

We can use MCP instead of the light bulb algorithm to get the following theorem. In this case the run time will improve by more than a logarithmic factor.

Theorem 4.1. We can find the pair $(i, j)$ of SNPs that maximizes $\left|P_{A}(i, j)-P_{B}(i, j)\right|$ in an expected time of $O\left(n^{1+\frac{\log p_{1}}{\log p_{2}} \log n}\right)$, where $p_{1}$ and $p_{2}$ are the smallest and the next smallest values of $\left|P_{A}(i, j)-P_{B}(i, j)\right|$, respectively, over all possible pairs $(i, j)$ of SNPs.
Proof: Similar to that of Theorem 3.1 and hence omitted. We call our algorithm for the two locus problem TLA.

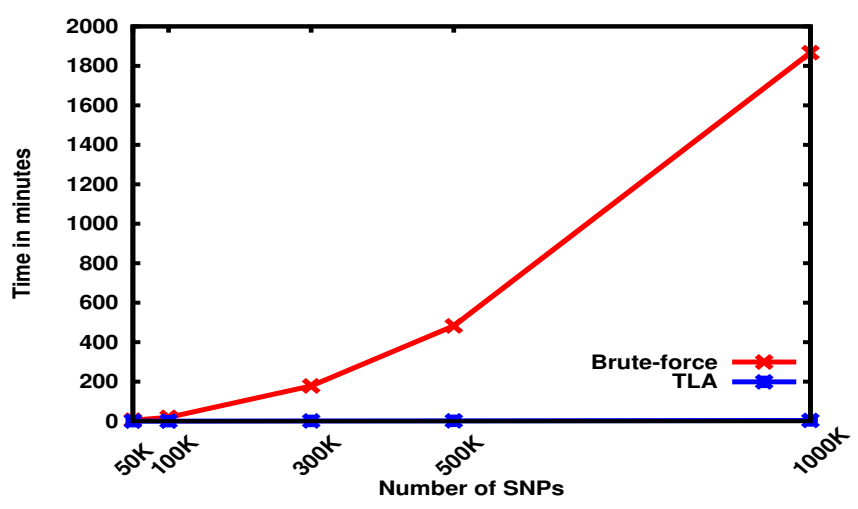

Figure 2: Performance evaluations between Brute-force and TLA methods.

\section{AN EXPERIMENTAL COMPARISON}

The notion of similarity (between two SNPs) used in [1] is Pearson's correlation coefficient. In this paper the similarity we use is based on the Hamming distance. We believe that the Hamming distance is a better measure since it can be used to derive other measures. In terms of run times, these two measures do not have a significant difference. Specifically, the complement of the Hamming distance is the measure of similarity we employ. The authors of [1] have tested their algorithms on different data sets (including random data). Since we do not have access to either these data sets or their algorithms, the only comparison we can do was on the random data. As explained in [1], we have also generated SNPs randomly. In particular, for each subject, the value of each SNP is chosen uniformly randomly to be either 0 or 1 with equal probability. This dataset is called NOISE Data in [1] (c.f. Table 4 in [1]). The authors of [1] note that random data are the hadest instances for the identification of the most (and least) correlated pair as well as the two locus problem. We have generated data of sizes $50 \mathrm{~K}, 100 \mathrm{~K}, 300 \mathrm{~K}, 500 \mathrm{~K}$ and $1,000 \mathrm{~K}$ with 50 cases and 50 controls to demonstrate how effective our algorithm is. For example FastANOVA [16] took almost 1000 seconds for $42 \mathrm{~K}$ SNPs. On the other hand for 50K SNPs our algorithm took only 7.8 seconds. All the experiments were done on an Intel Haswell compute node with 48 GB of RAM. The operating system running was Red Hat Enterprise Linux Server release 5.7 (Tikanga).

We have also compared the performance of our algorithm with the algorithm reported in [1]. Our results are shown in Table 4 . The recall rates for us are much better than those of [1] (see Table 6 in [1]). Recall rates refer to the accuracy of the different algorithms in terms of how many of the top $q$ correlated pairs were identified by these algorithms (for different values of $q$ ). For example, for $50 \mathrm{~K}$ SNPs, the top 10 recall rate of [1] is 0.2 , whereas it is 0.74 for our algorithm. As another example, for $100 \mathrm{~K}$ SNPs, the top 10 recall rate of [1] is 0 whereas it is 0.74 for our algorithm.

In terms of run times we can only do an estimation of the speedup since they [1] do not report any actual run times. They report that the brute force algorithm takes several days using 1,000 processors (for less than 1,000K SNPs). To give 
Table 4: Number of pairs and runtime comparisons in Hamming space on NOISE datasets. CPU times are given in minutes.

\begin{tabular}{lllllllll}
\hline & Brute-force & & TLA & & & & Gain & Speed up \\
\hline \# SNPs & Processed Pairs & CPU Time & Processed Pairs & CPU Time & Top 10 & Top 100 & Processed Pairs & Time \\
\hline $5 \times 10^{4}$ & $25 \times 10^{8}$ & 4.97 & 715,553 & 0.13 & 0.74 & 0.87 & $3,493.80$ & 39.29 \\
\hline $1 \times 10^{5}$ & $10 \times 10^{9}$ & 19.00 & $2,861,457$ & 0.22 & 0.74 & 0.92 & $3,494.72$ & 84.85 \\
\hline $3 \times 10^{5}$ & $90 \times 10^{9}$ & 178.23 & $25,749,044$ & 0.87 & 0.80 & 0.94 & $3,495.28$ & 203.96 \\
\hline $5 \times 10^{5}$ & $25 \times 10^{10}$ & 482.30 & $71,529,899$ & 1.35 & 0.88 & 0.83 & $3,495.04$ & 356.09 \\
\hline $1 \times 10^{6}$ & $10 \times 10^{11}$ & $1,867.05$ & $286,110,791$ & 3.48 & 0.80 & 0.75 & $3,495.15$ & 536.00 \\
\hline
\end{tabular}

the benefit of doubt, we take the time as 2 days. This means that for 100K SNPs, the brute force algorithm took 20 days on a single processor. For $100 \mathrm{~K}$ SNPs, in Table 6 (of [1]) they indicate that their algorithm achieves a speedup of 184 over the brute force. This means that their algorithm took more than 156 minutes. Also they have used 400 subjects whereas we have used 100 subjects. This means that on 100 subjects and $100 \mathrm{~K}$ SNPs, their algorithm will take 39 minutes. Our run time for $100 \mathrm{~K}$ SNPs and 100 subjects is 0.22 minutes. This means that our algorithm is more than 177 times faster. Note that this is only a very conservative estimate. Also note that the improvement our algorithm achieves is quite significant since the typical processing times reported in the literature for the two locus problem are quite high. The improvement we achieve is not only due to the novel algorithm but also due to a number of techniques we have used at the implementation level. We have used bit operations as much as possible. This also reduces the memory used. Any improvement in the run time achieved for solving the two locus problem could make a noticeable difference in GWAS. Please, see Figure 2 for visual details.

\section{CONCLUSIONS}

In this paper we have presented a novel algorithm for the two locus problem that plays a central role in GWAS. Our algorithm is two orders of magnitude faster than previous algorithms. We also offer algorithms for the finding the most and least correlated pairs of strings. In summary, we improve the results presented in many prior papers including [16] and [1].

\section{Funding}

This work has been supported in part by the following grants: NIH R01-LM010101 and NSF 1447711.

\section{REFERENCES}

[1] P. Achlioptas, B. Schölkopf and K. Borgwardt, Two-locus association mapping in subquadratic runtime, ACM SIGKDD international conference on Knowledge discovery and data mining (KDD) 2011.

[2] C.E. Aston, D.A. Ralph, D.P. Lalo, S. Manjeshwar, B.A. Gramling, D.C. DeFreese, A.D. West, D.E. Branam, L.F. Thompson, M.A. Craft, D.S. Mitchell, C.D. Shimasaki, J.J. Mulvihill, and E.R. Jupe, Oligogenic combinations associated with breast cancer risk in women under 53 years of age, Human Genetics, 116(3):208-221, Feb. 2005.

[3] M. S. Charikar. Similarity estimation techniques from rounding algorithms, Proc. ACM Symposium on Theory of Computing (STOC), 2002.
[4] B. Chiu, E. Keogh, and S. Lonardi, Probabilistic discovery of time series motifs, Proc. of the 9th International Conference on Knowledge Discovery and Data mining (KDD), pp. 493-498, 2003.

[5] J.H. Cho, D.L. Nicolae, L.H. Gold, C.T. Fields, et al., Identification of novel susceptibility loci for inflammatory bowel disease on chromosomes 1p, 3q, and 4q: evidence for epistasis between $1 p$ and IBD1, Proceedings of the National Academy of Sciences of the United States of America, 95(13):7502-7507, June 1998.

[6] T. H. Consortium, A second generation human haplotype map of over 3.1 million SNPs, Nature, 449(7164):851-61, Oct. 2007.

[7] H. J. Cordell, Detecting gene-gene interactions that underlie human diseases, Nat Rev Genet, 10(6):392-404, June 2009.

[8] N.J. Cox, M. Frigge, D.L. Nicolae, P. Concannon, C.L. Hanis, G.I. Bell, and A. Kong, Loci on chromosomes 2 (NIDDM1) and 15 interact to increase susceptibility to diabetes in mexican americans, Nature Genetics, 21(2):213-215, Feb. 1999.

[9] R. Motwani and P. Raghavan, Randomized Algorithms, Cambridge University Press, 1995.

[10] S. K. Musani, D. Shriner, N. Liu, R. Feng, et al., Detection of gene $\mathrm{x}$ gene interactions in genome-wide association studies of human population data, Human Heredity, 63(2):67-84, 2007.

[11] R. Nakamichi, Y. Ukai, and H. Kishino, Detection of closely linked multiple quantitative trait loci using a genetic algorithm, Genetics, 158(1):463-475, May 2001.

[12] R. Paturi, S. Rajasekaran, and J. Reif. The light bulb problem, Information and Computation 117, 1995, pp. 187-192.

[13] Y. Wang, X. Liu, K. Robbins, and R. Rekaya, AntEpiSeeker: detecting epistatic interactions for case-control studies using a two-stage ant colony optimization algorithm, BMC Bioinformatics, 3:117-117, 2010.

[14] J. Xu, C.D. Langefeld, S.L. Zheng, E.M. Gillanders, et al., Interaction effect of PTEN and CDKN1B chromosomal regions on prostate cancer linkage, Human Genetics, 115(3):255-262, Aug. 2004.

[15] X. Zhang, S. Huang, F. Zou, and W. Wang, TEAM: efficient two-locus epistasis tests in human genome-wide association study, Bioinformatics (Oxford, England), 26(12):i217-227, June 2010.

[16] X. Zhang, F. Zou, and W. Wang, Fastanova: an efficient algorithm for genome-wide association study, Proc. 14th ACM SIGKDD international conference on Knowledge discovery and data mining (KDD), pages 821-829, Las Vegas, Nevada, USA, 2008. 